\title{
Erratum to: Identification and management of patients at increased risk of osteoporotic fracture: outcomes of an ESCEO expert consensus meeting
}

\author{
J. A. Kanis ${ }^{1,2}$ - C. Cooper ${ }^{3,4}$ - R. Rizzoli ${ }^{5}$ - B. Abrahamsen ${ }^{6}$ - N. M. Al-Daghri ${ }^{7}$. \\ M. L. Brandi ${ }^{8}$. J. Cannata-Andia ${ }^{9}$ B. Cortet $^{10}$ - H. P. Dimai ${ }^{11}$ - S. Ferrari ${ }^{5}$ P. Hadji ${ }^{12}$. \\ N. C. Harvey ${ }^{3}$ - M. Kraenzlin ${ }^{13}$ • A. Kurth ${ }^{14,15}$ • E. McCloskey ${ }^{1,16}$ • S. Minisola ${ }^{17}$. \\ T. Thomas ${ }^{18}$ - J.-Y. Reginster ${ }^{19}$ • for the European Society for Clinical and Economic \\ Aspects of Osteoporosis, Osteoarthritis and Musculoskeletal Diseases (ESCEO)
}

Published online: 7 August 2017

(C) International Osteoporosis Foundation and National Osteoporosis Foundation 2017

\section{Erratum to: Osteoporos Int \\ DOI: 10.1007/s00198-017-4009-0}

The article "Identification and management of patients at increased risk of osteoporotic fracture: outcomes of an ESCEO

The online version of the original article can be found at http://dx.doi.org/ 10.1007/s00198-017-4009-0

\section{J. A. Kanis}

w.j.Pontefract@shef.ac.uk

1 Centre forMetabolic Bone Diseases, University of SheffieldMedical School, Beech Hill Road, Sheffield S10 2RX, UK

2 Institute for Health and Ageing, Catholic University of Australia, Melbourne, Australia

3 MRC Lifecourse Epidemiology Unit, University of Southampton, Southampton, UK

4 NIHR Musculoskeletal Biomedical Research Unit, University of Oxford, Oxford, UK

5 Service of Bone Diseases, Geneva University Hospitals and Faculty of Medicine, Geneva, Switzerland

6 Institute of Clinical Research, University of Southern Denmark, Odense, Denmark

7 Prince Mutaib Chair for Biomarkers of Osteoporosis, Biochemistry Department, College of Science, King Saud University, Riyadh, Saudi Arabia

8 Department of Surgery and Translational Medicine, Unit of Bone and Mineral Diseases, University of Florence, Florence, Italy

9 Bone and Mineral Research Unit, Instituto "Reina Sofía" de Investigación, REDinREN ISCIII, Hospital Universitario Central de Asturias, Universidad de Oviedo, Asturias, Spain expert consensus meeting", written by J.A. Kanis, C. Cooper, R. Rizzoli, B. Abrahamsen, N. M. Al-Daghri, M. L. Brandi, J. Cannata-Andia, B. Cortet, H. P. Dimai, S. Ferrari, P. Hadji, N. C. Harvey, M. Kraenzlin, A. Kurth, E. McCloskey, S. Minisola17, T. Thomas, and J.-Y. Reginster for the European

10 Department of Rheumatology, Lille University Hospital, Lille, France

11 Department of Internal Medicine, Division of Endocrinology and Diabetology, Medical University of Graz, Graz, Austria

12 Department of Bone Oncology, Endocrinology and Reproductive Medicine, Krankenhaus Nordwest, Frankfurt, Germany

13 Endonet, Endocrine Clinic and Laboratory, Basel, Switzerland

14 Department of Orthopaedic Surgery and Osteology, Klinikum Frankfurt, Frankfurt, Germany

15 Mayor Teaching Hospital, Charitè Medical School, Berlin, Germany

16 MRC and Arthritis Research UK Centre for Integrated Research in Musculoskeletal Ageing, Mellanby Centre for Bone Research, University of Sheffield, Sheffield, UK

17 Department of Internal Medicine and Medical Disciplines, "Sapienza" Università di Roma, Rome, Italy

18 INSERM U1059, Laboratoire Biologie Intégrée du Tissu Osseux, Rheumatology Department, CHU Saint-Etienne, Université de Lyon, Saint-Etienne, France

19 Department of Public Health, Epidemiology and Health Economics, University of Liège, Liège, Belgium 
Society for Clinical and Economic Aspects of Osteoporosis, Osteoarthritis and Musculoskeletal Diseases (ESCEO), was originally published Online First without open access. After publication in volume 28, issue 7, pages 2023-2034 the author decided to opt for Open Choice and to make the article an open access publication. Therefore, the copyright of the article has been changed to (c) The Author(s) 2017 and the article is forthwith distributed under the terms of the Creative Commons Attribution 4.0 International License (http://creativecommons.org/ licenses/by/4.0/), which permits use, duplication, adaptation, distribution and reproduction in any medium or format, as long as appropriate credit is given to the original author(s) and the source, a link to the Creative Commons license is provided, and any changes made are indicated. 